\title{
Functional abdominal complaints in pre-school children: parental reports of health-related quality of life
}

\author{
R. Oostenbrink $\cdot$ H. Jongman $\cdot$ J. M. Landgraf • \\ H. Raat $\cdot$ H. A. Moll
}

Accepted: 28 December 2009/Published online: 13 January 2010

(C) The Author(s) 2010. This article is published with open access at Springerlink.com

\begin{abstract}
Purpose The aim of this study is to assess the influence of functional abdominal complaints (FAC) on health-related quality of life in a group of Dutch pre-school children.

Methods Parents of children aged up to 6.0 visiting the outpatient pediatric department, Erasmus MC-Sophia, Rotterdam, The Netherlands in the period January 2005December 2006 for functional abdominal complaints during at least 3 months were asked to complete the Infant/ Toddler Quality of life Questionnaire (ITQOL), and questions of the abdominal pain index for use by parents to report pain symptoms in pre-school children. ITQOL scale scores of children with FAC were compared against with Dutch reference values. The abdominal pain index was tested for internal consistency and test-retest reliability. Correlations between ITQOL scale scores and abdominal pain index were assessed by Spearman's rank test.
\end{abstract}

R. Oostenbrink $(\square) \cdot$ H. Jongman

Outpatient Department Pediatrics, Erasmus MC-Sophia

Rotterdam, Dr Molewaterplein 60, room Sp 1549,

3015 GJ Rotterdam, The Netherlands

e-mail: r.oostenbrink@erasmusmc.nl

J. M. Landgraf

HealthActCHQ, Boston, MA, USA

e-mail: jml@healthactchq.com

H. Raat

Department of Public Health, Erasmus MC, Rotterdam,

The Netherlands

e-mail: h.raat@erasmusmc.nl

\section{H. A. Moll}

Outpatient Department Pediatrics, Erasmus MC-Sophia

Rotterdam, Dr Molewaterplein 60, room Sp 1540,

3015 GJ Rotterdam, The Netherlands

e-mail: h.a.moll@erasmusmc.nl
Results Results are based on 81 questionnaires completed by parents of children with FAC (response rate $61 \%$ ). Children had a median age of 46 months (interquartile range 27-59), 48\% girls. A significant impact was observed on most aspects of quality of life, particularly for physical functioning, general development, bodily pain, temperament and moods, general health perceptions and parental emotional impact. Parents of children with functional constipation tended to report lower scores than those of children with other FAC. The abdominal pain index appeared to be valid and was significantly correlated with ITQOL scales bodily pain and general health perceptions.

Conclusions A substantial lower health-related quality of life is reported in pre-school children with functional abdominal complaints, with effects on physical, emotional and parental domains. The 5-question severity index of abdominal pain appeared a valid tool and may be helpful to quickly assess the severity of abdominal pain in clinical practice.

Keywords Abdominal pain · Pre-school children · Health-related quality of life

\section{Introduction}

In general, functional abdominal complaints (FAC) have a frequency of about $10 \%$ in pediatrics and constitute about $3 \%$ of the outpatient pediatric visits [1,2]. Often these complaints lead to many investigations and long-term follow-up at the outpatient department, but a functional diagnosis without organic origin is determined for about 95\% of these cases [1-3]. In school-aged children and adolescents, FAC have shown to lead to social withdrawal and impaired physical ability in later life [4]. In addition, 
these complaints seem related to psychological factors in children and to parental distress and illness [2]. Studies on the health-related quality of life of children with FAC showed substantial effects when compared to healthy children [5, 6], indicating it is a serious condition with great influence on a child's life. Also in very young children, functional gastrointestinal disorders are frequently reported and may arise from maladaptive behavioral responses to stimuli or accompaniments to normal development. Complaints are defined based on a variable combination of chronic or recurrent symptoms not explained by structural or biochemical abnormalities [7, 8]. Specific studies investigating the effects of FAC in young children, however, are lacking. Measuring health-related quality of life in pre-school children is complicated by rapid changes in children's abilities overtime, as well as age-specific development and the need of rating by a proxy $[9,10]$. The Infant/Toddler Quality of Life questionnaire (ITQOL) is a generic health status measurement developed and validated for young children $[11,12]$. Such an instrument might also be augmented by additional pain-specific tools designed to assess the level of complaints in a standardized way. Walker et al. [13] has developed and validated the Pain Response Inventory to measure abdominal pain of the adolescents. We adjusted this Pain Response Inventory into the abdominal pain index (API) for use by parents to report pain symptoms in pre-school children. Together such instruments-general and specific-would be useful in evaluating the effects of treatment and fluctuation in complaints overtime.

The goal of our study was to assess the influence of functional abdominal complaints on health-related quality of life using ITQOL in a group of Dutch pre-school children. In addition, we validated the abdominal pain rating instrument (API) completed by parents.

\section{Materials and methods}

\section{Patients}

The study was performed at the Erasmus MC-Sophia, Rotterdam, The Netherlands at the outpatient department of General Pediatrics. Patients were prospectively included in the period January 2005-December 2006. Eligible patients were aged up to 72 months, visiting the pediatric outpatient with functional abdominal complaints during the past 3 months [7, 8], severe enough to interfere with daily activities, such as playing, eating, behavior and requiring medical treatment by a pediatrician. Parents were required to understand and read Dutch. Patients with a medical history suggestive for underlying pathology (history of necrotizing enterocolitis, Hirschsprung's disease, pyloric hypertrophy, short-bowel, cystic fibrosis, percutaneous endoscopic gastric catheter) or with specific symptoms suggestive for an underlying pathology (acute vomiting, gastrointestinal blood loss, fever, anorexia, weight loss, anemia) were excluded. Diagnostic tests to assess or to exclude underlying pathology were performed at the convenience of the doctor, but included bacterial and parasitic cultures, inflammatory markers and serology on celiac disease in children with specific symptoms as mentioned earlier. Patients were also excluded when they had a severe psychomotor retardation. Parents of eligible patients $(n=138)$ were asked to participate by a written invitation. On receipt of informed written consent $(n=99)$, they were mailed the study questionnaire. Reminding letters were sent by mail after 1 month. Response rate was $62 \%(n=85)$. To assess test-retest reliability of the abdominal pain score, a subset of 30 randomly selected patients was sent a shorter questionnaire within 4 weeks. The test-retest response rate was $67 \%(n=20)$. The Medical Ethics Committee of the Erasmus Medical Center has approved the study.

\section{Questionnaire}

General questions were asked on family situation, educational level, speaking and understanding the Dutch language and questions on chronic conditions like allergies, asthma, eczema, ear or other complaints. Next, we asked specific questions on the type of abdominal complaints (frequency of complaints, defecation pattern and localization), medication use (laxatives, spasmolytics, anti-emetics), and frequency of visits to a health care professional.

\section{Abdominal Pain Index}

In absence of a suitable objective measure for use by parents to report abdominal pain symptoms in pre-school children, we developed the Abdominal Pain Index (API) translated and derived from The Pain Response Inventory [13]. The Pain Response Inventory for children is originally developed and validated developed by Walker et al. [13] to measure abdominal pain in adolescents. The API for pre-school children aims to measure the severity of abdominal complaints and comprises five items assessing the frequency, duration and intensity of episodes of abdominal pain during the previous 4 weeks. Frequency of abdominal pain in past 4 weeks and the typical daily frequency are rated on a 6-point scale (none to every day/ constantly). The typical duration of pain episodes is rated on an 8-point scale (response categories including few minutes, about half an hour, about an hour, between 1 and $2 \mathrm{~h}, 3-4 \mathrm{~h}, 5-6 \mathrm{~h}$, most of the day, and all day). The typical and maximum intensity of abdominal pain in the past 4 weeks are rated using a 10-point scale ranging from no 
pain (0) to the most pain possible (10). Responses to the five pain ratings were standardized, and the sum was computed to yield an index of abdominal pain, with a higher value for more pain. The abdominal pain index was translated into Dutch according to international guidelines, including two independent forward translations and backward translation of one reconciled translation by a native English speaker [14].

\section{Infant and Toddler Quality of Life Questionnaire} $[15,16]$

The Infant and Toddler Quality of Life Questionnaire (ITQOL) is a generic health status measure for children aged 2 months up to 5 years, including items and scales to measure aspects of physical functioning, development, pain, mood, behavior, general health and impact on parents. The ITQOL consists of 97 items divided over 9 multiitem scales and two single-item questions. The items generally refer to the past 4 weeks. Following the ITQOL standard procedure, per scale, the items were summed up and transformed into a scale ranging from 0 (worst score) to 100 (best possible score). In accordance with specified scoring procedures, if a respondent missed $50 \%$ or more of items within a given scale, a score was not calculated. The Physical Functioning scales include a "not doing yet" option; responses as such were not included in the analysis. The General Behavior, Getting Along scale and Change in Health items refer to children aged 1 year and older. The ITQOL has been translated into Dutch with Dutch reference values available [16] and has been applied in various diseased populations in The Netherlands [17, 18]. Internal consistency was satisfactory with a Cronbach's alpha > 0.70 for all ITQOL scales in a sample of healthy children and a respiratory illness sample. Test-retest reliability was moderate (six ITQOL scales showing ICC 0.5-0.7) to adequate (four ITQOL scales showing ICC > 0.70) [16].

\section{Definitions}

Definitions of functional abdominal complaints were based on the Rome criteria for children $<4$ years [7] and for children $>4$ years [8]. In our study population, the following diagnoses were assessed based on the presence of symptoms and signs: irritable bowel syndrome (abdominal complaints related to change in frequency or change of appearance of defecation at least 1 time per week during past 2 months); functional abdominal pain (abdominal complaints with insufficient criteria to other FAC, at least 1 time per week during past 2 months); dyschezia (at least $10 \mathrm{~min}$ of straining and crying before successful passage of soft stools in normally healthy infants $<6$ months); functional constipation [at least 2 of the following during
1 month (for children $>4$ years during 2 months)]: 2 or fewer stools per week, excessive stool retention, painful or hard bowel movements, scybalous, pebble like or hard stools for a majority of stools, or 1 period of secondary fecal incontinence; all complaints in absence of evidence of structural, endocrine, inflammatory or metabolic disease.

Analysis

Respondents not at all or little able to read and understand Dutch, as judged using information of the questionnaire on a scale of 'not at all'_-little'_-'fairly' — 'good', were excluded from the analysis $(n=4)$. We expected significantly lower scores in children with FAC compared to reference values for the ITQOL Physical Functioning and Bodily Pain scales. A smaller but still significantly effect was expected for scales including Growth and Development, Temperament and Moods, Parental Emotional Impact and Time Impact. Non-parametric tests were used to assess significant differences because skewed data were observed for ITQOL scale scores in our study. ITQOL scale scores observed in the present sample were compared to Dutch reference values [16] using Mann-Whitney $U$ test. In addition, within-group differences in ITQOL scores were examined for children with functional constipation versus those with other FAC. Effect sizes were calculated to determine the clinical significance of observed differences. Effect sizes relate differences in mean scores between subgroups to the dispersion of the scores ((Mean (general population) - Mean (RAP population))/standard deviation (largest of the two). Effect sizes $(d)$ were defined following Cohen's guidelines [19]: small effect $0.2 \leq d<0.5$; moderate effect $0.5 \leq d<0.8$ and large effect $d \geq 0.8$. Effect sizes of 0.5 are considered clinically relevant.

Since we applied the Abdominal Pain Index (API) for use by parents to report abdominal pain symptoms in preschool children in this study for the first time, we evaluated ceiling and floor effects (i.e. $>25 \%$ of responders reporting the highest or lowest response category) and internal consistency by using Cronbach's alpha for the standardized API. Test-retest reliability was assessed using intra class coefficients: $>0.70$ was considered adequate [20]. Additionally, at the group level, test-retest reliability was assessed by paired sample $t$-test, and by effect sizes for significant test-retest differences [21].

Correlations between ITQOL scale scores and the API were assessed by Spearman's rank test. In particular, we expected correlations between the API score and ITQOL Bodily Pain scale. To a lesser extent, correlations might be present for domains sensitive to pain, e.g., physical Functioning, general health perceptions and temperament and moods. 


\section{Results}

The study sample was comprised of 81 patients with FAC (39 girls; 48\%), with a median age of 46 months, not different from non-responders $(50 \%$ girls, median age 40 months (25th-25th percentile 28-54). Demographic characteristics are described in Table 1. Reported chronic conditions other than recurrent abdominal complaints included asthma-like symptoms $(n=22)$, ear complaints $(n=20)$, allergies $(n=21)$, eczema $(n=16)$ and others $(n=4)$. Medication prescription for FAC included one or more laxatives in 78 and anti-emetics in two cases; spasmolytics were never prescribed at all. Children with and without constipation were comparable for age, and hospitalization frequency, but children with constipation were significantly more boys (54 vs. 44\%), and reported more frequently other comorbidity (57 vs. $32 \%$ ), visits to the pediatrician (4.3 vs. 1.9) and medication use (98 vs. $90 \%$ ) than those with other abdominal complaints.

Twenty-nine parents of children did not report on the items included in the abdominal pain index (API) due to absence of abdominal pain in the last 4 weeks. Three parents did not respond to one or more of the items in the API, resulting in 49 valid observations of the API. The overall API score did not show floor or ceiling effects, but a floor effect was observed for the sub-item frequency of abdominal pain (26\% reporting the lowest category). Mean observed value for API was -0.19 with SD 3.77 (range -7.76-8.62). Internal consistency was good (Cronbach's alpha 0.79). Test-retest analysis is based on 20 subjects, with a mean of 52 days (SD 20) between responses. Test-retest reliability was moderate (ICC 0.64). We did observe significant testretest differences neither for the overall API (mean difference -1.58, SD 3.4) nor for the separate API items.

Table 2 presents the ITQOL scale scores as observed in children with FAC. There was little missing data $(0-4 \%)$ at the item level for some ITQOL scales, which values were left out from the analysis. Physical functioning items pertaining to a certain minimal level of development/age were answered by 'not doing yet' in $>50 \%$ by two respondents. Compared to the reference population, significantly lower scores were reported in our population with FAC on 7 out of 11 ITQOL scales (Table 2). A large effect size was observed for the Growth and Development scale (0.84), and moderate effect sizes were observed for Physical Functioning, Bodily Pain, Temperament and Moods, General Health Perceptions and the Parental Emotional Impact (range 0.56-0.74) scales. Parents in our sample reported their child's current health to be substantially better relative to the previous year (mean score for Change in Health 65.6). The mean observed for the Change in Health item in the FAC sample was significantly higher
Table 1 General characteristics of study population with functional abdominal complaints $(n=81)$

\begin{tabular}{|c|c|}
\hline \multicolumn{2}{|l|}{ Respondent } \\
\hline Woman & $71(88 \%)$ \\
\hline Age $(\text { years })^{\mathrm{a}}$ & $34.6(5 \%)$ \\
\hline \multicolumn{2}{|l|}{ Employment } \\
\hline Employed & $58(72 \%)$ \\
\hline Housemaker & $14(17 \%)$ \\
\hline Unemployed/disabled & $9(11 \%)$ \\
\hline \multicolumn{2}{|l|}{ Highest educational level } \\
\hline Elementary school & $1(1 \%)$ \\
\hline Secondary school & $50(62 \%)$ \\
\hline Higher educational/university & $29(36 \%)$ \\
\hline Two parents' household & $72(89 \%)$ \\
\hline \multicolumn{2}{|l|}{ Child } \\
\hline \multirow[t]{3}{*}{ Age (months) } & Median: 46 \\
\hline & $\begin{array}{l}\text { 25th-75th percentile: } \\
27-59\end{array}$ \\
\hline & Range: $5-72$ \\
\hline Girls & $39(48 \%)$ \\
\hline \multicolumn{2}{|l|}{ Classification of abdominal pain } \\
\hline Irritable bowel syndrome & $10(12 \%)$ \\
\hline Functional abdominal pain & $7(9 \%)$ \\
\hline Dyschezia & $1(1 \%)$ \\
\hline Functional constipation & $63(78 \%)$ \\
\hline $\begin{array}{l}\text { Parent reported chronic condition other than } \\
\text { abdominal }\end{array}$ & $41(51 \%)$ \\
\hline \multicolumn{2}{|c|}{ Frequency abdominal complaints during past 4 weeks $^{\text {b }}$} \\
\hline Most days-every day & $19(25 \%)$ \\
\hline 4-12 days & $14(17 \%)$ \\
\hline$<4$ days & $21(26 \%)$ \\
\hline \multicolumn{2}{|l|}{ Pain frequency per day during past 4 weeks $^{\mathrm{b}}$} \\
\hline Whole day & $9(11 \%)$ \\
\hline $4-6$ times a day & $9(11 \%)$ \\
\hline $1-3$ times a day & $31(28 \%)$ \\
\hline No pain & $4(5 \%)$ \\
\hline \multicolumn{2}{|l|}{ Duration of pain in past 4 weeks ${ }^{\mathrm{b}}$} \\
\hline Most of the day & $10(12 \%)$ \\
\hline $1-4 \mathrm{~h}$ & $12(15 \%)$ \\
\hline $30 \mathrm{~min}$ & $18(22 \%)$ \\
\hline Few minutes & $13(16 \%)$ \\
\hline \multicolumn{2}{|l|}{ Location of abdominal complaints ${ }^{\mathrm{b}}$} \\
\hline Upper abdomen & $5(6 \%)$ \\
\hline Umbilical & $24(30 \%)$ \\
\hline Lower abdomen & $35(43 \%)$ \\
\hline Divers & $9(11 \%)$ \\
\hline Abdominal pain index ${ }^{\mathrm{a}}$ & $-0.2(3.8)$ \\
\hline \multicolumn{2}{|c|}{ Primary care visits for abdominal complaints in past 12 months } \\
\hline 0 & $9(11 \%)$ \\
\hline $1-3$ & $45(56 \%)$ \\
\hline $4-12$ & $26(32 \%)$ \\
\hline
\end{tabular}


Table 1 continued

\begin{tabular}{ll}
\hline$>12$ & $1(1 \%)$ \\
$\begin{array}{l}\text { Specialist visits for abdominal } \\
\text { complaints in past } 12 \text { months }^{\mathrm{a}}\end{array}$ & $3.7(4.2)$ \\
$\begin{array}{l}\text { Hospitalization for abdominal } \\
\text { complaints in past } 12 \text { months }\end{array}$ & $6(7 \%)$ \\
Medication use & $78(96 \%)$ \\
\hline a Mean (SD) & \\
b Data not reported by all parents &
\end{tabular}

than the control sample score (score 56.1). Parents of children with constipation reported a lower score on the scale General Health Perceptions (score 63.0, SD 19.2) scale compared to parents of children with other FAC. Although not statistically significant, lower scores on all other ITQOL scales were observed for the constipation sample.

As expected, significant correlations were observed for the ITQOL Bodily Pain scale and the API (Spearman's rho $-0.39, P<0.01)$ and for the ITQOL General Health Perceptions scale and API (Spearman's rho $-0.32, P=0.03$ ). Corrected for the presence of comorbidity, these correlations remained similar. For all other ITQOL scale scores, negative associations with the API were observed but not significant.

\section{Discussion}

In a Dutch sample of pre-school children with functional abdominal complaints, we observed substantial lower scores on most ITQOL domains, with moderate to large effect sizes. Not only pain domains but also physical, emotional and general domains of quality of life are affected. Children with constipation report more frequently medical consumption and report lower ITQOL scores compared to children with other FAC. Lower health-related quality of life in children with constipation may be explained by the high medical consumption (high frequency of specialist visit and medication prescription). The abdominal pain index for Dutch pre-school children showed well feasibility ( $88 \%$ response), no floor or ceiling effects with good internal consistency and moderate test-retest reliability. Improved scaling of pain, using the abdominal pain index, may help in monitoring treatment effects. This may support future use of this measure in clinical practice for a severity index of abdominal pain. The correlation between API score (higher pain scores relate to lower ITQOL scores) and ITQOL scales Bodily Pain and General Health Perceptions is conform our expectations, as both these scales address physical complaints. These findings provide further empirical evidence of the multidimensional construct validity of the ITQOL.

Studies in older children with FAC or defecation disorders have shown substantial affected health-related quality in life on functional, behavioral and emotional domains $[5,6,22]$. These results largely agree with our observations. The effects of FAC on social and physical dysfunction in later life [4] are often explained by the wide range of quality of life domains that are affected in children with FAC. The effect of reduced quality of life during childhood on adult functioning may even be stronger in the rapidly developing young child and needs to be considered in the management of young children with abdominal complaints. As observed in previous studies in infants with a chronic condition [17, 18], higher scores on the item Change in Health were observed for children with FAC compared to reference values. This

Table 2 Mean ITQOL scores (SD) for children with FAC compared to the reference values

\begin{tabular}{llll}
\hline ITQOL scale & FAC & Reference values [16] & ${\text { Effect size }(\mathrm{d})^{\mathrm{a}}}^{\mathrm{a}}$ \\
\hline Physical functioning $(n=79)$ & $86.9(15.8)$ & $97.2(9.8)$ & 0.65 \\
Growth and development $(n=81)$ & $75.4(13.2)$ & $86.5(10.6)$ & 0.84 \\
Bodily pain $(n=81)$ & $66.6(23.6)$ & $83.8(16.8)$ & 0.74 \\
Temperament and moods $(n=80)$ & $69.2(12.7)$ & $77.2(10.5)$ & 0.63 \\
General behavior $(n=75)^{\mathrm{b}}$ & $71.0(14.0)$ & $72.8(12.7)$ & $71.4(8.8)$ \\
Getting along $(n=73)^{\mathrm{b}}$ & $73.0(9.0)$ & $79.0(14.5)$ & 0.71 \\
General health perceptions $(n=81)$ & $65.7(18.7)$ & $92.1(10.5)$ & 0.56 \\
Parental impact: emotion $(n=81)$ & $83.3(15.7)$ & $93.0(11.0)$ & 0.30 \\
Parental impact: time $(n=80)$ & $88.4(15.5)$ & $75.3(18.8)$ & -0.37 \\
Family cohesion $(n=80)$ & $75.9(21.7)$ & $56.1(18.4)$ & \\
Change in health $(n=72)^{\mathrm{b}, \mathrm{c}}$ & $65.6(26.0)$ & \\
\hline
\end{tabular}

${ }^{a}$ For differences with $P<0.05$ only

b 6 Children aged $<1$ year were not included in scale scores General Behaviour, Getting Along and Change in Health

${ }^{c}$ A score of 50 indicates a similar perceived health as 1 year ago, scores higher and lower than 50 indicate a better or worse perceived health, respectively 
finding is not unexpected given the nature of FAC in young children (sometimes accompaniments to normal development [7]) and the general good health of most infants/toddlers that is not expected to change remarkably over the course of a year. Another explanation for this finding is that Change in Health may refer to a preference answer and more frequently applies to a diseased population than to a healthy one. Therefore, this scale is more useful in repetitive measures in one diseased population than in the comparison of two independent groups. Neither the child's age nor the duration of abdominal complaints was related to scores on Change in health, which would be expected if this improvement resulted from new recently treated patients being included in our study.

We used the effect size to interpret the clinical significance of differences of ITQOL scale scores. Previous studies using ITQOL in other disease populations $[17,18]$ show effect sizes ranging from 0.3 to 1.2 , which values largely agree with the observations in this study. Similar to our observations, previous studies $[12,17,18]$ report the impact of clinical conditions on ITQOL domains Physical Functioning, General Health Perceptions and Parental Impact. The large effect of FAC on ITQOL domain Bodily Pain seems to be related to the characteristics of this condition in particular.

As might be expected, several limitations to the study are worth noting. First, although the population was sufficiently large to detect a 7.5-point difference in scale scores between the disease population and reference values (effect size 0.5 with SD 15), the diversity of functional abdominal complaints was limited. Our population of children with FAC comprised a majority of children with constipation. Therefore, our study sample did not allow subgroup analysis for ITQOL domain scores. Second, as our FAC sample was slightly older than the reference population (mean 24 months, SD 12.3 months), this may have affected the differences in scale scores between the two groups. However, further analysis in this regard showed that age and ITQOL scale scores are independent from one another. Finally, inherent to measuring quality of life in pre-school children, parents were asked to proxy-report for their young children. Proxy ratings may be confounded by several factors $[23,24]$ and often underestimate psychological domains $[9,10,25]$.

In conclusion, functional abdominal complaints in preschool children are related to substantial lower reported health-related quality of life as measured by the ITQOL. In addition to pain, FAC affect a broad range of physical, emotional a nd general aspects of health. Finally, the API with five questions appears to be a valid tool for measuring abdominal pain and may be helpful to quickly assess abdominal complaints in clinical practice.
Open Access This article is distributed under the terms of the Creative Commons Attribution Noncommercial License which permits any noncommercial use, distribution, and reproduction in any medium, provided the original author(s) and source are credited.

\section{References}

1. Loening-Baucke, V. (1993). Constipation in early childhood: Patient characteristics, treatment, and longterm follow up. Gut, 34, 1400-1404.

2. Ramchandani, P. G., Hotopf, M., Sandhu, B., \& Stein, A. (2005). The epidemiology of recurrent abdominal pain from 2 to 6 years of age: Results of a large, population-based study. Pediatrics, $116,46-50$.

3. Loening-Baucke, V. (2005). Prevalence, symptoms and outcome of constipation in infants and toddlers. The Journal of Pediatrics, 146, 359-363.

4. Hotopf, M., Carr, S., Mayou, R., Wadsworth, M., \& Wessely, S. (1998). Why do children have chronic abdominal pain, and what happens to them when they grow up? Population based cohort study. British Medical Journal, 316, 1196-1200.

5. Youssef, N. N., Murphy, T. G., Langseder, A. L., \& Rosh, J. R. (2006). Quality of life for children with functional abdominal pain: A comparison study of patients' and parents' perceptions. Pediatrics, 117, 54-59.

6. Faleiros, F. T., \& Machado, N. C. (2006). Assessment of healthrelated quality of life in children with functional defecation disorders. Journal of Pediatrics (Rio J), 82, 421-425.

7. Hyman, P. E., Milla, P. J., Benninga, M. A., Davidson, G. P., Fleisher, D. F., \& Taminiau, J. (2006). Childhood functional gastrointestinal disorders: Neonate/toddler. Gastroenterology, 130, 1519-1526.

8. Rasquin, A., Di Lorenzo, C., Forbes, D., Guiraldes, E., Hyams, J. S., Staiano, A., et al. (2006). Childhood functional gastrointestinal disorders: Child/adolescent. Gastroenterology, 130, 1527-1537.

9. Theunissen, N. C., Vogels, T. G., Koopman, H. M., Verrips, G. H., Zwinderman, K. A., Verloove-Vanhorick, S. P., et al. (1998). The proxy problem: Child report versus parent report in healthrelated quality of life research. Quality of Life Research, 7, 387-397.

10. Warschburger, P., Landgraf, J. M., Peterman, F., \& Freidel, K. (2003). Health-related quality of life in children assessed by their parents: Evaluation of the psychometric properties of the CHQPF50 in two German clinical samples. Quality of Life Research, 12, 291-301.

11. Klassen, A. F., Landgraf, J. M., Lee, S. K., Barer, M., Raina, P., Chan, H. P. W., et al. (2003). Health related quality of life in 3 and 4 year old children and their parents: Preliminary findings about a new questionnaire. Health and Quality Life Outcomes, $1,81$.

12. Klassen, A. F., Lee, S. K., Raina, P., Chan, H. W., Matthew, D., \& Brabyn, D. (2004). Health status and health-related quality of life in a population-based sample of neonatal intensive care unit graduates. Pediatrics, 113, 594-600.

13. Walker, L. S., Smith, C. A., Garber, J., \& van Slyke, D. A. (1997). Development of the pain response inventory for children. Psychological Assessment, 9, 392-405.

14. Guillemin, F., Bombardier, C., \& Beaton, D. (1993). Cross-cultural adaptation of health-related quality of life measures: Literature review and proposed guidelines. Journal of Clinical Epidemiology, 46, 1417-1432.

15. Landgraf, J. M. (1994). The Infant/Toddler quality of life questionnaire: Conceptual framework, logic, content and preliminary psychometric results. Final Report to Schering-Plough 
Laboratories and Health Technology Associates. Boston: New England Medical Center.

16. Raat, H., Landgraf, J. M., Oostenbrink, R., Moll, H. A., \& EssinkBot, M. L. (2007). Reliability and validity of the Infant and Toddler Quality of Life Questionnaire (ITQOL) in a general population-and respiratory disease sample. Quality of Life Research, 16, 445-460.

17. Oostenbrink, R., Jansingh-Piepers, E. M., Raat, H., Nuijsink, M., Landgraf, J. M., Essink-Bot, M. L., et al. (2006). Health-related quality of life of pre-school children with wheezing illness. Pediatric Pulmonology, 41, 993-1000.

18. Oostenbrink, R., Spong, K., de Goede-Bolder, A., Landgraf, J. M., Raat, H., \& Moll, H. A. (2007). Parental reports of healthrelated quality of life in young children with neurofibromatosis type 1: Influence of condition specific determinants. The Journal of Pediatrics, 151, 182-186.

19. Cohen, J. (1977). Statistical power analysis for the behavioral sciences. New York: Academic Press.
20. Bland, J. M., \& Altman, D. G. (1997). Cronbach's alpha. British Medical Journal, 314, 572.

21. Andresen, E. M., Catlin, T. K., Wyrwich, K. W., \& JacksonThompson, J. (2003). Retest reliability of surveillance questions on health related quality of life. Journal of Epidemiology and Community Health, 57, 339-343.

22. Benninga, M. A., Voskuijl, W. P., Akkerhuis, G. W., Taminiau, J. A., \& Buller, H. A. (2004). Colonic transit times and behaviour profiles in children with defecation disorders. Archives of Disease in Childhood, 89, 13-16.

23. Rosenbaum, P. L., \& Saigal, S. (1996). Measuring health-related quality of life in pediatric populations: Conceptual issues. In B. Spilker (Ed.), Quality of life and pharmacoeconomics in clinical trials (pp. 785-791). Philadelphia: Lippincott-Raven Publishers.

24. Eiser, C. (1997). Children's quality of life measures. Archives of Disease in Childhood, 77, 350-354.

25. Addington-Hall, J., \& Kalra, L. (2001). Who should measure quality of life? British Medical Journal, 322, 1417-1420. 